\title{
Evaluación y experiencias en el control de conificación en pozos con alto corte de agua en yacimientos con empuje hidráulico
}

\author{
Ramos $^{1 *}$ L., Marín, A. ${ }^{2 * *}$ \\ $1^{*}$ Miembro SPE, Consultor Independiente, Bogotá, Colombia \\ ${ }^{2 * *}$ Miembro SPE, Consultor Independiente y Soporte Técnico Externo SNF SA, Buenos Aires, Argentina \\ *E-mail: ramoslu9616@yahoo.com \\ **E-mail: amaurymt@icloud.com
}

\begin{abstract}
Resumen
La producción de petróleo con alta relación agua-petróleo (RAP) en yacimientos con fuerte empuje de agua de fondo, generalmente se manifiesta en los pozos productores de petróleo y gas como un mecanismo de conificación. Principalmente, el avance del contacto agua-petróleo (CAP) se mueve hacia los intervalos abiertos a producción en el tope de la arena productora. Este tipo de mecanismo o problema es difícil de diagnosticar y corregir. Un diagnóstico limitado y desconocimiento de la ventana de aplicación de tecnologías para mitigar dicho mecanismo, han demostrado ser un factor importante de la baja tasa de éxito para solucionar dicho problema. En este artículo se presentan ciertas consideraciones o criterios con base a la experticia de los autores, para el adecuado diagnóstico del mecanismo de conificación de agua, así como, se revisan y sugieren las soluciones químicas, mecánicas e híbridas más prospectivas, a la fecha.
\end{abstract}

Palabras clave: RAP (relación agua petróleo), conificación de agua, CAP (contacto agua petróleo), tecnologías control de agua, acuíferos activos.

\section{Assessment and expertise insights on coning control for watered out wells in reservoirs with aquifer support}

\begin{abstract}
Oil production with high water-oil ratio (WOR) in strong water drive reservoirs are prone to generate coning in watered-out production wells. The water-oil contact (WOC) moves upwards through the open perforated intervals in the top of producer sand. Water coning problems are difficult to diagnose and to correct. An inadequate diagnosis and poor knowledge regarding the window of application of technological solutions to control water coning, have evidenced to be an important factor of the low success rate in solving this problem. This paper presents insights based on author's expertise, for the precise diagnostics of water coning, and the most prospective technical solutions among chemicals, mechanicals and hybrids methods to date.
\end{abstract}

Keywords: WOR (water oil ratio), water coning, WOC (water oil contact), water control technologies, active aquifers.

\section{Introducción}

La producción excesiva de agua representa uno de los problemas más críticos durante la explotación de yacimientos de petróleo y gas, especialmente en yacimientos maduros y en proyectos CEOR ("Chemical Enhanced Oil Recovery”). En muchos casos, el corte de agua sobrepasa el $90 \%$ afectando las etapas siguientes del proceso y manejo de la producción de fluidos.

En el diagnóstico de los posibles mecanismos o problemas de alta producción de agua, es muy común en la práctica revisar la información básica disponible y de uso común, como son la historia de producción

Cita: Ramos, L. y Marín, A. (2018). Evaluación y experiencias en el control de conificación en pozos con alto corte de agua en yacimientos con empuje hidráulico. Revista Fuentes: El reventón energético, vol 16(2), 23-40. 
(graficas de tasas y relación agua-petróleo vs tiempo), registros resistividad/GR ("resistivity/gamma ray logs") y de cementación, gráficas de diagnóstico tipo Chan o Ramos y correlaciones estratigráficas entre pozos vecinos. El análisis e integración de esta información, permite una primera aproximación del diagnóstico y así definir la estrategia, selección y diseño de soluciones adecuadas para la prevención y/o corrección del tipo de problema existente. En la mayoría de los casos, este diagnóstico se puede ver afectado por la carencia o escasa de esa información y además a las incertidumbres asociadas de las herramientas que se utilicen para el análisis. Es importante destacar, que el proceso de diagnóstico para identificación del mecanismo o problema de alta producción de agua requiere de tiempo y adicionalmente los costos asociados, pueden ser bastante altos. En muchos casos, esto representa una limitante muy importante en la compañía que opera el campo, yacimiento o pozo, al impactar negativamente y precisamente en el adecuado proceso de diagnóstico del mecanismo de producción de agua. Soliman, Rester, Sigal, Everett y Johnson (2000) resumieron resultados de 900 casos de control de producción de agua. Este estudio reporta que el beneficio económico de un tratamiento sin el adecuado diagnóstico estaba en el orden de USD 85 por cada dólar invertido. Sin embargo, con el diagnóstico y tratamiento apropiado, el beneficio económico se incrementó a USD 562 por cada dólar invertido

Existen varias causas para la producción excesiva de agua y por lo general se dividen en problemas en las cercanías del pozo o "near-wellbore" (p. e. fugas del revestidor, flujo con y sin restricciones detrás de la tubería relacionados con la calidad de la cementación, etc.) y problemas propios del yacimiento o "reservoirrelated", tales como conificación y canalizaciones por zonas de alta permeabilidad, entre otras (Seright, Lane \& Sydansk, 2001) (Jaripatke \& Dalrymple, 2010). En los casos donde el mecanismo de producción es por empuje hidráulico de fondo (acuífero), el problema de producción de agua se manifiesta, en la gran mayoría de los casos, como una conificación para pozos productores verticales o conocido como "cusping o cresting" en los pozos horizontales. Este último no será parte del presente trabajo.

El mecanismo de conificación se puede definir como la entrada de agua de fondo al pozo ocasionado por las altas tasas de producción. Estas altas tasas, permiten un desbalance del equilibrio dinámico presente en un contacto de agua/petróleo subyacente y que existe previamente a la puesta en producción del pozo productor (Muskat, 1949). Este desequilibrio dinámico influye también sobre fuerzas viscosas asociadas con el gradiente de presión descrito por la ley de Darcy (Martin, 2017) y relacionadas con el flujo de fluidos denle el yacimiento, así como sobres fuerzas gravitacionales que actúan en dirección vertical y causan que un fluido ascienda por la diferencia de densidad respecto a otro, haciendo todo esto que este mecanismo sea complejo.

En base a diversas experiencias de diferentes operadores, se logran identificar algunas deficiencias prácticas operativas, que además junto al desconocimiento del uso o de la ventana de aplicación de las soluciones tecnológicas para controlar el mecanismo de la conificación de agua, ha demostrado ser parte importante de la baja tasa de éxito para solucionar este problema.

En el presente trabajo se discuten diversos criterios y consideraciones para hacer un adecuado diagnóstico del problema de alta producción de agua por el mecanismo de conificación de agua, así como la visualización e identificación de las posibles soluciones para mitigar dicho problema.

\section{Mecanismos que inciden en la producción excesiva de agua}

Inicialmente, es indispensable comprender las características del yacimiento y los mecanismos de recuperación (p.e. primaria y secundaria) (Satter \& Thakur, 1994) (Thakur \& Satter, 1998). Respecto a los mecanismos de recuperación primaria los principales son expansión de líquido y roca, gas en solución, expansión por capa de gas, empuje por agua, y segregación gravitacional (Satter \& Thakur, 1994). De estos, el empuje por agua es el que aporta mayor recuperación de los fluidos del yacimiento. La presencia de acuíferos activos es característica de este mecanismo de producción y se debe resaltar que el influjo de agua puede ser lineal, radial y no simétrico (Velandia, 2013). Por sus condiciones de borde, los acuíferos se clasifican como finito e infinito. Cuando en un yacimiento existe la presencia de casquetes o capas de gas y de acuíferos activos, se pueden obtener los beneficios de diferentes mecanismos de producción mencionados con anterioridad. En estos casos la eficiencia de recobro puede ser bastante alta con estrategias óptimas de gerencia de yacimiento.

Por otra parte, los procesos de recobro secundario consisten en tecnologías que ayudan a mejorar la recuperación de los hidrocarburos por encima del mecanismo de recobro primario y evitar la abrupta declinación de producción. Los métodos más usuales son los de inyección de agua e inyección de gas, y los mismos representan estrategias de mantenimiento de 
presión del yacimiento. En ambos casos, la posible rápida irrupción del agua o del gas, en los pozos productores puede evidenciarse debido a diversos factores (p.e. heterogeneidad de yacimiento, altas tasas de extracción o "drawdown", presencia de fallas o fracturas y canales de alta permeabilidad o zonas ladrona, entre otras).

Independientemente de los procesos de recobro secundario y en presencia de acuíferos activos, se puede dar el caso de que la producción de petróleo ocurra con un exceso de producción de agua en superficie. De ocurrir este evento, la producción excesiva de agua generalmente es definida como agua indeseada ("bad water" / "unwanted water") (Bailey et al., 2000). Si la producción de agua está afectando el recobro del hidrocarburo, claramente se puede diagnosticar por el método grafico de la relación agua petróleo (RAP o WOR del inglés "Water Oil Ratio”) versus la producción acumulada de petróleo (Np) también conocida como las curvas WOR vs. Np, (Prado, Palencia, Reyna, Fernández, Espinosa \& Rauseo, 2005). Sin embargo, este método de diagnóstico será discutido más adelante. Independientemente de que un yacimiento particular este en la etapa de recobro primario o secundario, la producción de agua indeseada genera altos costos operativos, impactos ambientales y reducción de la productividad de aceite con altos cortes de agua (Reyes, Ortiz \& Azuaje, 2010). En la Figura 1 se muestra la distribución característica de los costos promedio del "ciclo cerrado del agua" que incluyen los gastos de capital o CAPEX (del inglés Capital Expenditures) y gastos operacionales u OPEX (del inglés Operating Expenditures) (Khatib \& Verbeek, 2002). Estos costos generalmente se incrementan con el tiempo, sin embargo, resulta de gran interés conocer la distribución típica de estos costos en un campo bajo recobro secundario.

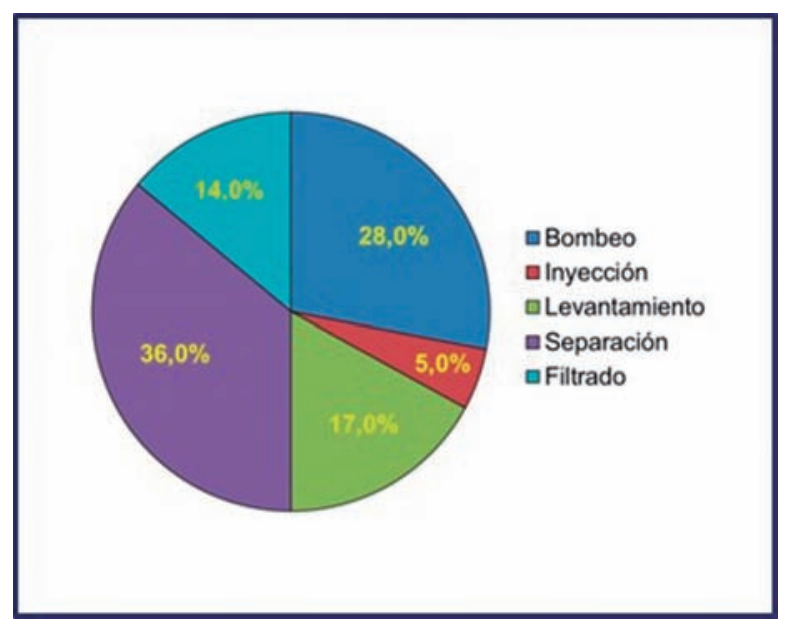

Figura 1. Distribución de costos promedio del ciclo cerrado del agua (modificado de Khatib \& Verbeek, 2002).
A continuación, se procede a clasificar los factores principales asociados a la producción de agua en exceso en dos categorías, de acuerdo con la fuente del problema donde se ha generado: en la cercanía del pozo y a nivel de yacimiento (Jaripatke \& Dalrymple, 2010). Respecto a los posibles problemas de producción de agua en las cercanías del pozo, algunos de los problemas reportados comúnmente incluyen:

- Fugas en el revestidor o "casing",

- Canalización detrás del revestidor,

- Daños causados por acumulación de incrustaciones o bacterias,

- Pozo completado con perforaciones cercanas a las zonas de agua,

- Completación en zonas de transición de fases agua/ petróleo.

En cualquiera de estos casos, se puede observar un incremento abrupto del corte de agua en el tiempo. En este caso, es importante que durante la etapa de diagnóstico se identifique(n) el (o los) punto(s) de ingreso del agua en el pozo productor, con el fin de evaluar posibles estrategias de control de agua que apliquen al caso evaluado.

Para el caso de los problemas de producción de agua a nivel de yacimiento, que puede incluir zonas que van más allá del área de drenaje del pozo productor, los problemas más comunes reportados incluyen pero no se limitan las siguientes condiciones:

- Conificación,

- Canalización en zonas de alta heterogeneidad o altos contrastes de permeabilidad,

- Adedamiento por alta relación de movilidad,

- Zonas de alta conductividad como fracturas y/o fallas.

Usualmente, la irrupción del agua hacia las perforaciones del pozo ocurre en el lapso donde se aprecia un aumento abrupto o gradual del corte de agua o relación agua-petróleo (RAP). Esta RAP puede llegar a fases de estabilización o continuar incrementándose hasta alcanzar el límite económico permitido antes de la posible intervención, cierre o abandono del pozo. Para el caso específico del presente trabajo, el objetivo principal se enfocará específicamente en el diagnóstico y posibles soluciones para el caso de conificación de agua (Figura 2). 


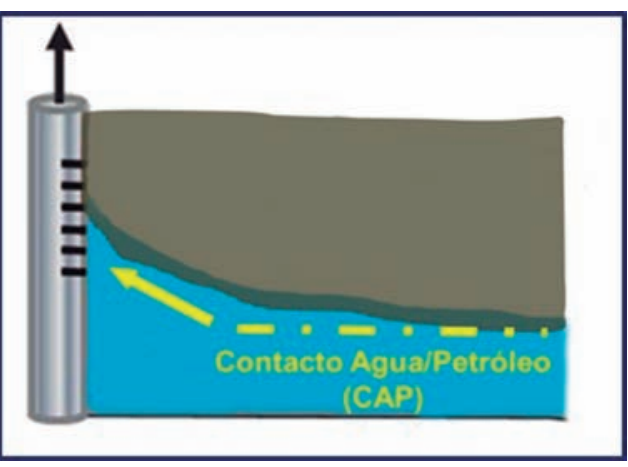

Figura 2. Conificación de agua en pozo vertical (modificado de Sydansk \& Romero-Zerón, 2011).

Este caso especial ha sido solucionado parcialmente por muchos operadores de forma convencional, sin mucho criterio técnico o buscando una solución rápida, económica y poco eficaz. La práctica común ha sido el cierre del pozo para abatir el cono en su ápice, y completar el pozo con perforaciones en la parte superior de la arena (en el ático, lejos del contacto agua-petróleo o CAP).

Es importante destacar que la conificación es un caso muy particular que se presenta en los yacimientos con empuje por agua, si ocurren las siguientes condiciones que se mencionan a continuación: alta relación de permeabilidades $\left(\mathrm{k}_{\mathrm{v}} / \mathrm{k}_{\mathrm{h}}\right)$, alta relación de movilidad, presencia de un acuífero de fondo activo (sin importar su geometría y condiciones de borde), pozo completado en cercanía al contacto agua-petróleo (CAP), y producción de fluidos por encima de la tasa critica de conificación. La combinación de todos los factores mencionados aumentara el grado de la conificación, que se traduce en una rápida irrupción del agua indeseada hacia los perforados del pozo.

Sin entrar en detalles de cómo hacer el estimado de la tasa critica de conificación para pozos verticales en yacimientos anisotrópicos, a continuación se presenta correlación o método analítico donde se resalta que de todas las condiciones mencionadas en el párrafo anterior, específicamente la relación $\left(\mathrm{k}_{\mathrm{v}} / \mathrm{k}_{\mathrm{h}}\right)$ resulta ser la más determinante en el efecto de conificación del agua desde el CAP hacia los intervalos perforados del pozo vertical. Este método analítico fue desarrollado por Chaperon (1986) y consiste en desarrollar cálculos en tres pasos como se describe a continuación:

$$
\begin{gathered}
q_{o}=\frac{4.888 \times 10^{-4}}{B_{o}} \frac{h^{2} k_{h}}{\mu_{o}} \Delta \rho q_{c}^{*} \\
q_{c}^{*}=\frac{0.7311}{\left(\frac{1.9434}{\alpha}\right)}
\end{gathered}
$$

$$
\alpha=\frac{r_{e}}{h} \sqrt{\frac{k_{v}}{k_{h}}}
$$

Donde se indican las dimensiones a continuación:

$\mu_{o}$ : viscosidad de aceite, $\mathrm{cP}$

$q_{o}$ : tasa critica de aceite sin conificación, barriles estándar por día (STB/d)

$h$ : espesor de la columna de aceite, pies

Bo: factor volumétrico de formación de aceite, barriles de yacimiento por barriles estándar (RB/STB)

$\Delta \rho$ : gradiente de densidad entre las fases aceite y agua, $\mathrm{g} / \mathrm{cc}$

$r_{e}$ : radio de drenaje, pies

$k_{v}$ : permeabilidad vertical, md

$k_{h}$ : permeabilidad horizontal, md

Esta correlación es válida para pozos de penetración total, y se asume que el espesor del intervalo perforado es "h". Hay que resaltar que hay otras correlaciones para pozos de penetración parcial, sin embargo, este tópico en particular esta fuera del alcance y objetivos del presente trabajo.

Por otra parte, el mecanismo de conificación también se puede validar a partir de simulación numérica. Kuo \& DesBrisay (1983) y Kuo (1989) analizaron el desarrollo de la conificación por medio de simulación considerando el efecto de cuatro parámetros importantes del yacimiento: relación de permeabilidades $\mathrm{k}_{\mathrm{v}} / \mathrm{k}_{\mathrm{h}}$, (desde 0.01 a 1.0), penetración parcial del pozo (desde 20 a $80 \%$ de espesor de la columna de aceite), tasa de producción (desde 500 a 2000 BFPD), y relación de movilidad (desde 1.0 a 10). En orden de importancia, y haciendo análisis de sensibilidad con esos parámetros, la relación de permeabilidades $\mathrm{k}_{\mathrm{v}} / \mathrm{k}_{\mathrm{h}}$, seguido de la relación de movilidad (M), representan parámetros críticos en el mecanismo y/o desarrollo de la conificación en pozos verticales en yacimientos anisotrópicos, con presencia de acuíferos activos.

En la siguiente sección se discutirán las posibles soluciones para los casos de conificación de agua por diferentes efectos (o sus posibles combinaciones) mencionados en la presente sección.

\section{Diagnóstico del problema de alta producción de agua asociado al mecanismo de conificación}

El diagnóstico del problema de alta producción de agua es un aspecto crítico y de suma importancia dada la diversidad de variables que pueden causar la conificación de agua. Elphick \& Seright (1998), Hardy 
\& Lockhart (1998), Halliburton (2001) y Jaripatke \& Dalrymple (2010) han reportado diversos tipos de problemas y jerarquizados por su complejidad en casos de estudio de conificación de agua. Por lo tanto, el uso adecuado de las técnicas, tecnologías y herramientas de diagnóstico, son muy importantes para disminuir las posibles incertidumbres asociadas con la identificación del tipo de problema y mecanismos de conificación de agua en un caso de estudio.

Una de las principales fallas en el diagnóstico del problema de conificación de agua es la incertidumbre asociada a la caracterización del yacimiento y la limitación en el conocimiento de la procedencia del agua (Soliman, East \& Gorel, 1999) (Seright, Lane \& Sydansk, 2001). Adicionalmente, la experiencia del proceso de diagnóstico en las compañías operadoras en conjunto con los proveedores de tecnologías para el control o solución del problema, ha demostrado que:

- El proceso de diagnóstico requiere tiempo y es costosa,

- La información del yacimiento y principalmente la del pozo bajo estudio, en muchas ocasiones puede ser incompleta y dispersa.
En este sentido, una guía para realizar un diagnóstico inicial, radica en la revisión, análisis e integración (RAI), de la información básica disponible y de uso común del sistema yacimiento-pozo, para así definir de manera definitiva si el problema del pozo en estudio es de conificación de agua. Desde la experiencia práctica de los autores, la Figura 3 presenta un diagrama sugerido del proceso RAI respecto a la información básica requerida y/o herramientas de diagnóstico de mayor uso, donde se minimizan los tiempos de RAI, son de bajo costo y que han demostrado ser muy útiles para la definición preliminar de las características y severidad del problema de conificación de agua. En caso de no estar definida la problemática con esta RAI inicial y tal como se observa en la misma Fig. 3 , se pueden incorporar herramientas más avanzadas y de mayor complejidad para complementar dicho diagnóstico.

A continuación, se resumen cada uno de los componentes del proceso de revisión-análisisintegración (RAI) sugerido, y con el fin de contribuir a definir el diagnóstico del mecanismo de alta producción de agua.

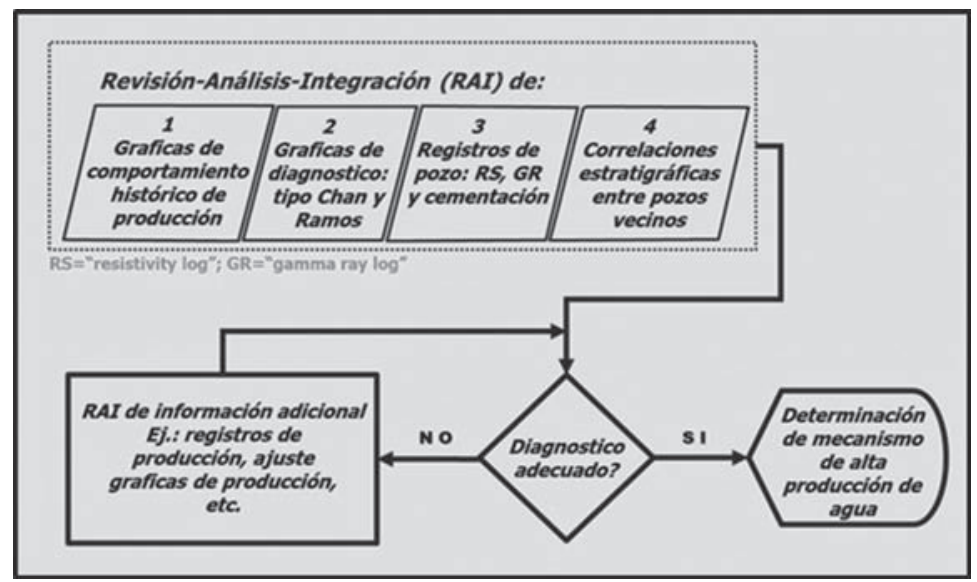

Figura 3. Diagrama del proceso de revisión-análisis-integración (RAI) para el diagnóstico del mecanismo de alta producción de agua.

\section{a) Análisis simultáneo de gráficas históricas de producción, recuperación y declinación}

En la práctica, la primera fuente de información y más inmediata del proceso de diagnóstico, son las gráficas históricas de:

- Producción; tasas de fluidos producidos, aceite (Qo), agua (Qw), y gas (Qg) vs. tiempo, t,

- Declinación de producción de petróleo: tasa de petróleo, Qo vs. producción acumulada, Np,
- Recuperación: relación agua-petróleo, RAP vs. producción acumulada, $\mathrm{Np}$.

Utilizando estas gráficas, Bailey et al., (2000) proponen una estrategia sencilla de análisis simultáneo de las mismas. Adicionalmente, en esta metodología se debe definir anticipadamente la relación agua-petróleo límite, con base al conocimiento del campo/yacimiento y donde el impacto económico de producir el pozo a ese nivel no sea rentable. Seguidamente se resume las diferentes etapas y su aporte con el proceso de diagnóstico. 
Cuando la tasa de petróleo comienza a disminuir al mismo tiempo que aumenta la tasa de producción de agua, representa un claro indicio que la mayor producción de agua está afectando el comportamiento de la producción de petróleo. En la Figura 4 se presenta un ejemplo de pozo con muy buena cementación influenciado por un acuífero de fondo. El comportamiento de la sección $\mathbf{A}$ está sugiriendo un avance paulatino o normal de agua que podría ser asociado al avance del acuífero o del contacto agua-petróleo. Estos avances "normales" de agua combinado con la declinación de producción de petróleo, en muchas ocasiones sugieren los inicios de un fenómeno de conificación. Posteriormente, en el comportamiento de la sección B se nota un aumento sustancial en la producción de agua que ocasiona un impacto en la producción de petróleo. Este último comportamiento es muy común cuando el tope del cono alcanza las perforaciones abiertas a producción, ocasionando una canalización temprana de agua.

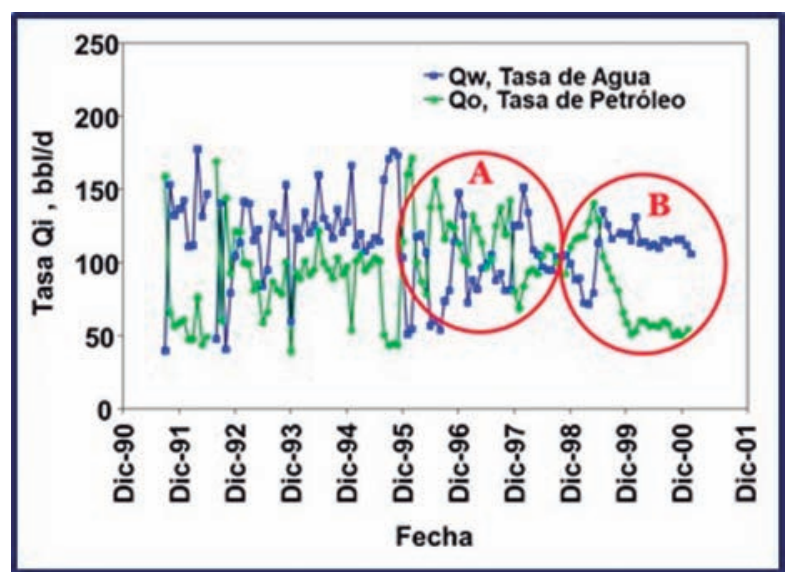

Figura 4. Comportamiento de producción, tasas de los fluidos producidos [barriles por día] vs tiempo (modificado según la estrategia de Bailey et al., 2000).

Otra manera de identificar potenciales problemas de alta producción de agua, es a partir de la evaluación de los cambios en la pendiente de la gráfica semi-logarítmica de tasa de petróleo, Qo vs. la recuperación acumulada de petróleo, Np; comúnmente llamada curva de declinación (Figura 5). Para este análisis es importante conocer con anticipación la tasa límite de producción de petróleo y en la cual se obtendría el Recobro Final Estimado o EUR (del inglés "Estimated Ultimate Recovery"), con el fin de identificar cuando ya no es económico producir el pozo. Adicionalmente, conocer esta tasa límite de Qo permite estimar las reservas recuperables del pozo en evaluación. En la Figura 5 se muestra ejemplo del impacto de los cambios en las tasas de producción de agua $(\mathrm{Qw})$ en el comportamiento de la declinación de producción de petróleo (Bailey et al., 2000).

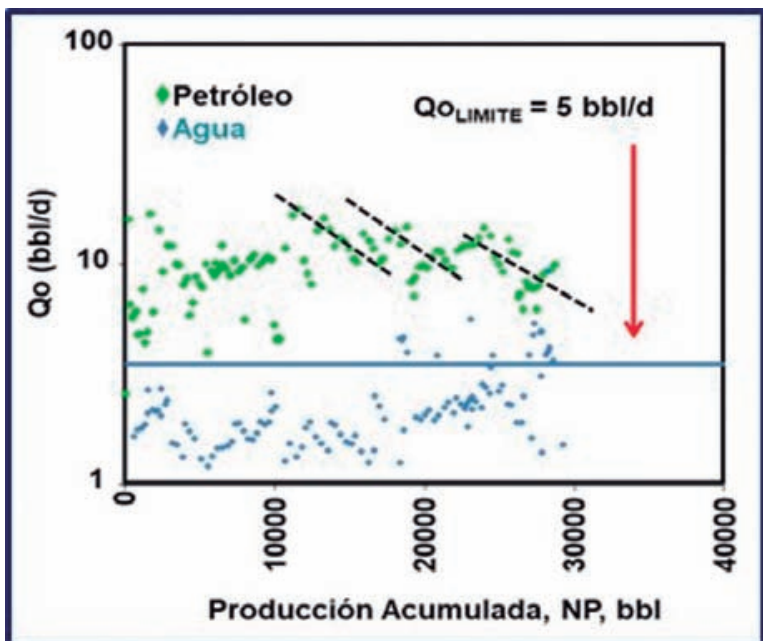

Figura 5. Comportamiento de la declinación de producción de petróleo (Modificado según la estrategia de Bailey et al., 2000).

La Figura 6, muestra la gráfica semi-logarítmica de recuperación representada por la relación agua-petróleo, RAP vs. la producción acumulada de petróleo, NP. Esta gráfica permite determinar las reservas de petróleo estimadas extrapolando la curva de recuperación hasta el valor de la RAP límite y compararlas con las reservas esperadas del pozo (comúnmente determinadas previamente a partir de la gráfica de declinación para estimar el EUR). Con esta comparación se puede estimar el potencial de pérdidas de reservas recuperables debido a los efectos de las altas tasas de producción de agua.

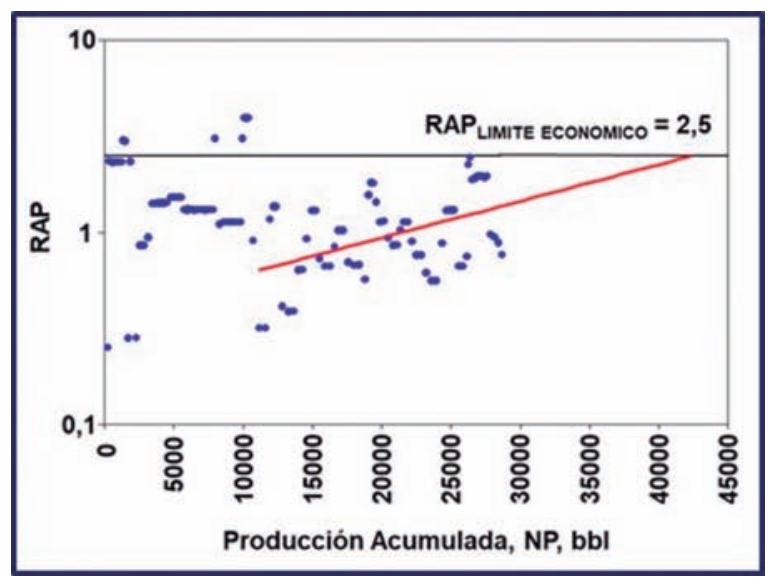

Figura 6. Comportamiento de recuperación: relación aguapetróleo (log RAP) vs. producción acumulada de petróleo, NP, (modificado según estrategia de Bailey et al., 2000).

\section{b) Gráficas de Diagnóstico de Chan}

El análisis de problemas de conificación de agua mediante el uso de las llamadas gráficas de diagnóstico (Chan, 1995) (Chan, Bond, Keese \& Lai, 1996) representa la 
primera opción gráfica para estimar o determinar el tipo de problema de alta producción de agua. Chan (1995) basado en estudios de simulación numérica, sugirió que a partir de la forma del gráfico log-log de la derivada de la relación agua-petróleo (RAP o WOR) vs. el tiempo acumulado de producción, se logran identificar las tendencias características para diferentes mecanismos de producción de agua tales como la canalización y la conificación. (Figura 7). Adicionalmente, este método puede emplearse para la evaluación de producción de agua producto de la combinación de diferentes factores a nivel de pozo y yacimientos, tales como las relaciones de permeabilidades vertical-horizontal $\left(\mathrm{k}_{\mathrm{v}} / \mathrm{k}_{\mathrm{h}}\right)$ y de movilidad (M), que son las principales variables que influencian la generación o desarrollo del mecanismo de conificación de agua.
Basados en estudio de simulación, Seright (1997) discute si el método de Chan puede efectivamente diferenciar los fenómenos de canalización y conificación de agua. De acuerdo a esta investigación, donde se evalúan diferentes condiciones de yacimiento y pozo, se concluye que los problemas de canalización multicapa de agua pueden ser interpretados incorrectamente como un problema de conificación de agua. Por lo tanto, para la evaluación de posibles de mecanismos de alta producción de agua usando las gráficas de diagnóstico de Chan se recomienda integrar y validar estos resultados con otros métodos y herramientas de diagnóstico.
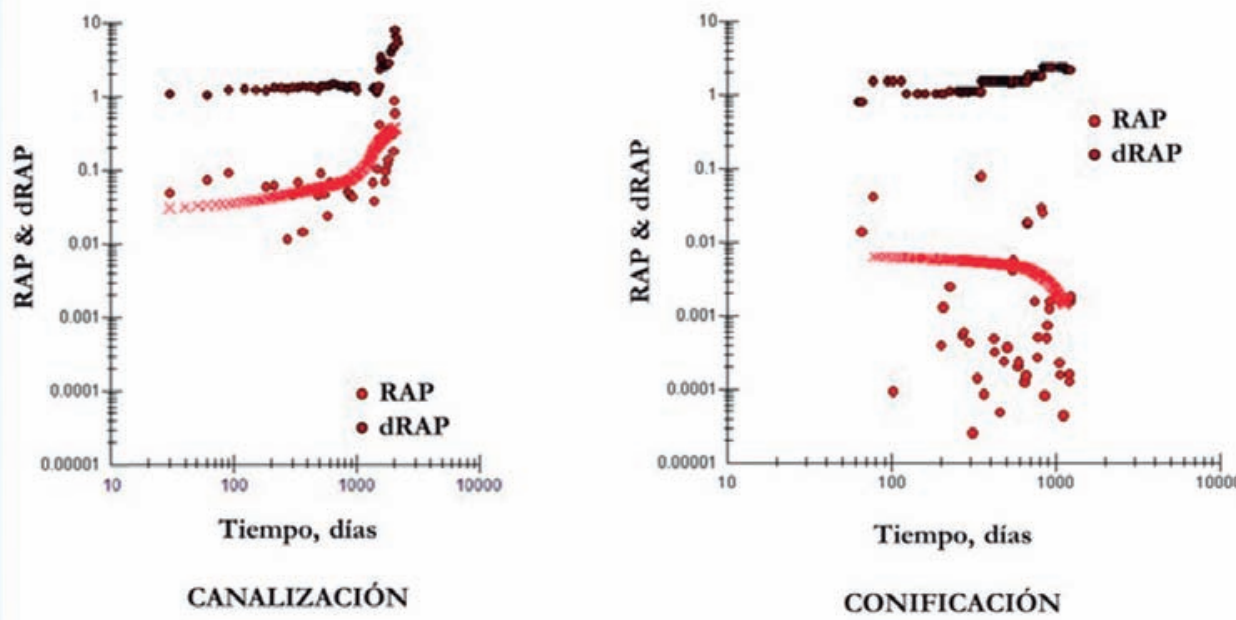

CONIFICACIÓN

Figura 7. Gráficas de Diagnostico Tipo: Canalización Multicapa y Conificación (modificado según el método de Chan, 1995).

\section{c) Curva Diagnóstico de Ramos}

Debido a la ineficiencia observada en el tratamiento de datos dispersos de las relaciones agua-petróleo (RAP) y la derivada de los mismos usando las curvas de diagnóstico de Chan, Ramos (2000) desarrolló un método analítico usando datos confiables de casos históricos. Con este método se logró validar la diferencia entre los mecanismos de conificación y canalización de agua.

El procedimiento del método consta de dos pasos (Ramos 200). Un primer paso, que es el ajuste potencial de la gráfica de producción acumulada de agua $\left(W_{p}\right)$ con respecto a la producción acumulada de petróleo $\left(N_{p}\right)$. De este ajuste se obtendrá la producción de petróleo al momento de la irrupción de agua $\left(N_{p B T}\right)$ :

$$
W_{p}=\frac{a}{(n+1) q_{a v}}\left(N_{p}-N_{p B T}\right)^{n+1}
$$

Donde,

$\mathrm{a}=$ coeficiente del ajuste de la gráfica por función de potencia (obtenido de la mejor regresión $\mathrm{R}^{2}$ de la función), $q_{a v}=$ tasa promedio de producción de petróleo,

$\mathrm{n}$ = exponente del ajuste potencial de $W_{p}=\mathrm{f}\left(N_{p}\right)$ (obtenido de la mejor regresión $\mathrm{R}^{2}$ de la función).

El segundo paso, corresponde a computar la RAP como una derivada de la función de potencia:

$$
R A P=\frac{d W_{p}}{d N_{p}}=\frac{a}{q_{a v}}\left(N_{p}-N_{p B T}\right)^{n}
$$


Finalmente, se construye la gráfica de RAP calculado por la Ecuación $\mathbf{5}$ vs. el tiempo ajustándola potencialmente:

$$
R A P=a^{\prime}\left(t-t_{b t}\right)^{m}
$$

Donde $a^{\prime}$ es el coeficiente del ajuste de la gráfica RAP, $t$ el tiempo, $t_{b t}$ es el tiempo en el que ocurre la irrupción de agua y $m$ representa el exponente que resulta del ajuste de esta gráfica usando la mejor regresión $\mathrm{R}^{2}$ de la función. Si el exponente $m$ en menor a la unidad ( $m<$ 1), el mecanismo de producción es el resultado de una conificación de agua.

Tanto Ramos (2000) como Llamedo, Mejías, González, Espinoza \& Valero (2005), han verificado el funcionamiento del procedimiento, al usarlo en casos reales de campo de pozos con problemas de conificación de agua y empleando la secuencia de las ecuaciones mencionadas (4, 5 y 6). Este método resulta simple de graficar utilizando herramientas convencionales (p.e. Excel).

\section{d) Otros Métodos Gráficos Sugeridos}

Entre algunos de los métodos de análisis y diagnóstico de utilidad para evaluar problemas de producción de agua se pueden mencionar los siguientes:

- Economides, Watters, Dunn-Norman, Hardy \& Lockhart (1996) determinan que en algunas ocasiones se pueden evidenciar inconvenientes durante la interpretación de las gráficas de corte de agua (BSW) vs. tiempo (Figura 8).

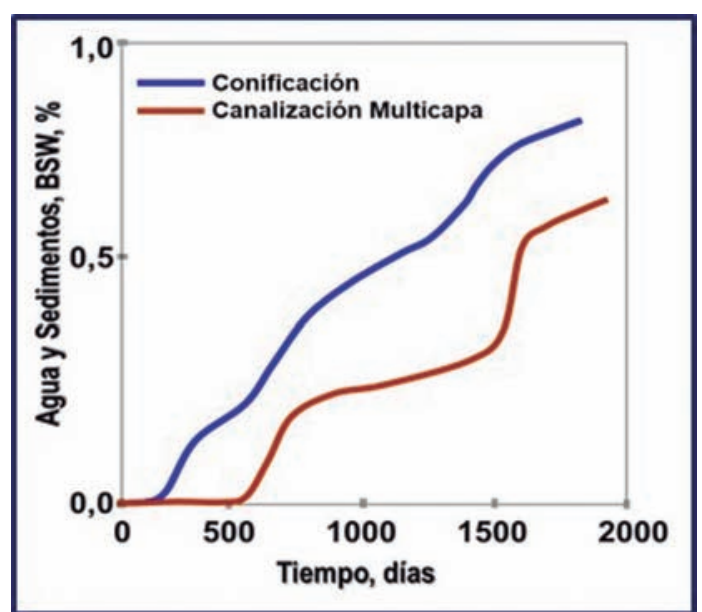

Figura 8. Comportamiento corte de agua (\% BSW) vs. tiempo (modificado de Economides et al., 1996).

- Recham, Osisanya \& Touami (2000) usando simulación numérica, desarrollan un análisis extensivo de diversas correlaciones empíricas junto con la posterior determinación de parámetros relevantes (yacimientos y fluidos) que influyen en la conificación de agua. Uno de los resultados relevantes de este estudio es que tanto la anisotropía vertical o perfil de heterogeneidades, $\mathrm{k}_{\mathrm{v}} / \mathrm{k}_{\mathrm{h}}$ (Figura 9) y relación de movilidades, M (Figura 10), representan las variables de mayor importancia que influyen en la de conificación de agua. Se destaca claramente que a mayores $\mathrm{k}_{\mathrm{v}} / \mathrm{k}_{\mathrm{h}}$ (Figura 9) y M (Figura 10) la producción de agua se acelera drásticamente en el tiempo. Los resultados de este estudio confirman los resultados reportados por Kuo \& DesBrisay (1983) y Kuo (1989).

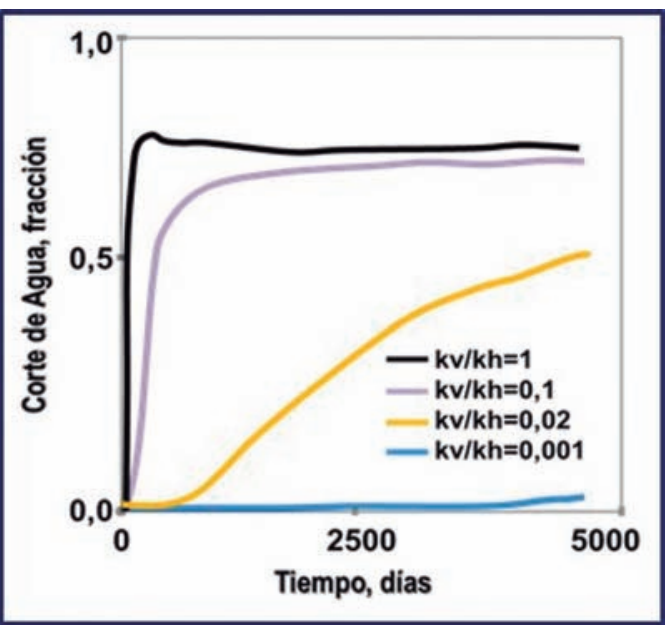

Figura 9. Efecto de la anisotropía vertical $\left(\mathrm{k}_{\mathrm{v}} / \mathrm{k}_{\mathrm{h}}\right)$ en el corte de agua (modificado de Recham et al. 2000).

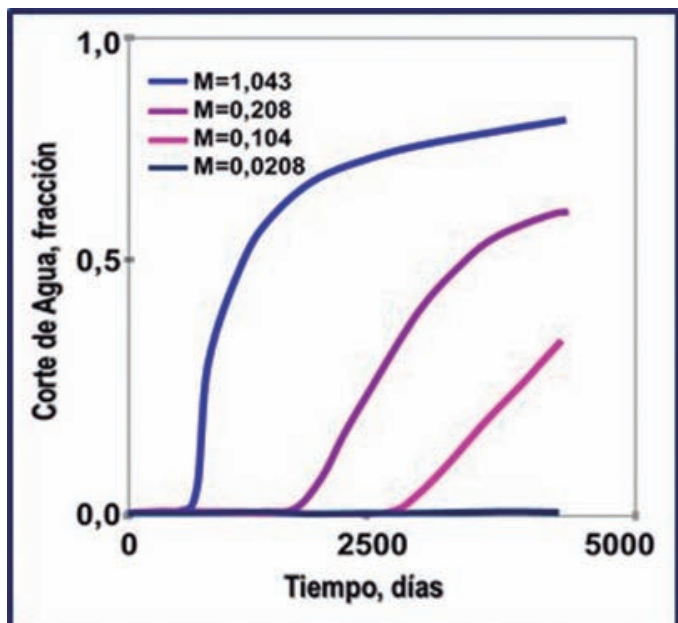

Figura 10. Efecto de la razón de movilidades M sobre el corte de agua (modificado de Recham et al. 2000).

- Zabala et al. (2017) presentan un modelo muy amplio de diagnóstico sobre el impacto de la producción excesiva de agua. En el mismo analizan exhaustivamente los comportamientos 
de los históricos de producción e inyección y las gráficas de diagnóstico convencionales complementadas con información de registros. Además proponen funciones para los comportamientos observados y así diagnosticar mecanismos como la conificación de agua en pozos productores. Este modelo es más detallado y complementa lo propuesto o sugerido en este trabajo, denominado proceso RAI.

\section{e) Análisis con registros de pozo}

Los registros de pozo que son corridos cuando el pozo no se ha completado, son los de hueco abierto ("open hole logs"), o cuando ya se ha completado y cementado ("cased hole logs"). En la clasificación de registros de hueco abierto, hay una gama variada de estos para detectar el nivel inicial del contacto aguapetróleo (CAP). El de mayor interés y uno de los de más común uso, es el registro eléctrico que incluya dos pistas ("tracks") que muestren la información registrada de rayos gamma o GR ("gamma ray”), de potencial espontáneo o SP (“spontaneous potential”) y de resistividad, versus la profundidad medida en MD ("Measured Depth") o en profundidad vertical real o TVD ("True Vertical Depth") en la zona de interés del pozo. En la curva de resistividad del registro eléctrico, se detecta el CAP si se aprecia un cambio súbito de baja hacia alta resistividad, justo donde se ubique el punto de inflexión de la curva. Es relativamente fácil de visualizar el contacto en las arenas de gran espesor con pocas intercalaciones de lutitas. Hay que enfatizar que este contacto será el inicial en la etapa productiva del pozo, es decir, este contacto no es estático en el tiempo y se moverá hacia la parte superior de la arenisca productora, de acuerdo con el empuje suministrado por el acuífero y tasa de extracción establecida para el pozo productor. Este análisis es válido para pozos verticales o con poca desviación.

Para el caso de los pozos altamente desviados y horizontales que navegan en zonas de poco espesor, pero con presencia de acuíferos activos de fondo, en el escenario que no sea factible detectar el CAP será necesario disponer adicionalmente del perfil de temperatura, para detectar las posibles entradas de agua hacia el pozo. Si hay presencia de "cusping" de agua, el punto de ingreso del agua de formación en la zona horizontal del pozo corresponderá a una temperatura elevada. El registro de resistividad también puede ser otra opción si existe el contraste suficiente entre el agua de producción y el crudo de la formación. Alrededor de ese punto de ingreso, es que se formara la cresta o “cusping”. Sin embargo, los casos de alta producción de agua en pozos horizontales o altamente desviados no serán discutidos en el presente trabajo.

Adicionalmente, en los pozos completados con revestidor es indispensable conocer la integridad del cemento, antes de sugerir cualquier solución de tipo mecánica o química para solventar el problema de conificación de agua. Con ayuda del registro para evaluar la calidad de la cementación conocido como CBL (del inglés “cement bond log”), se determina si existe un correcto aislamiento hidráulico entre la formación y el cemento (Halliburton, 2001). El registro de CBL consta de tres pistas. En la primera se dispone de las curvas de tiempo de viaje, GR y "Collar Locator", en la segunda se dispone de las curvas de amplitud, y en la tercera se dispone de la curva de micro sismograma ("Micro Seismogram"). La herramienta del CBL tiene un transmisor acústico y dos receptores. La señal acústica generada viaja a través del revestidor, cemento y la formación. El sistema de registro determina el tiempo de viaje y de amplitudes de la señal emitida por los receptores. El analista interpreta las señales de las curvas de los "tracks", y puede determinar si hay condiciones de: tubería libre, no se evidencia adherencia del cemento a la formación, cemento parcialmente aislado de la formación, y buena adherencia del cemento a la formación.

En aquellos casos que se detecte la condición de tubería libre, mala adherencia del cemento a la formación y otras anomalías como fugas detrás del revestidor, o presencia de micro-anillos, el pozo candidato deberá ser sometido a un trabajo de forzamiento con cemento, antes de proceder con la posible solución idónea para mitigar el problema de conificación de agua. En la Figura 11 se muestra un registro compuesto típico integrado por el CBL/VDL ("Cement Bond Log” / "Variable Density Log”) e información básica del registro eléctrico. El mismo indica una buena integridad del cemento en la zona de interés en la parte superior de la arena desde 4030 pies MD. Este pozo se encuentra perforado en el intervalo de 4030-4036 pies MD.

Finalmente, la integración del análisis de las curvas de diagnóstico e historia de producción de fluidos con la evaluación histórica de registros de pozos (desde la perforación y completación hasta sus fases de producción) contribuirán a desarrollar una interpretación adecuada de los posibles mecanismos de la conificación de agua de formación. 


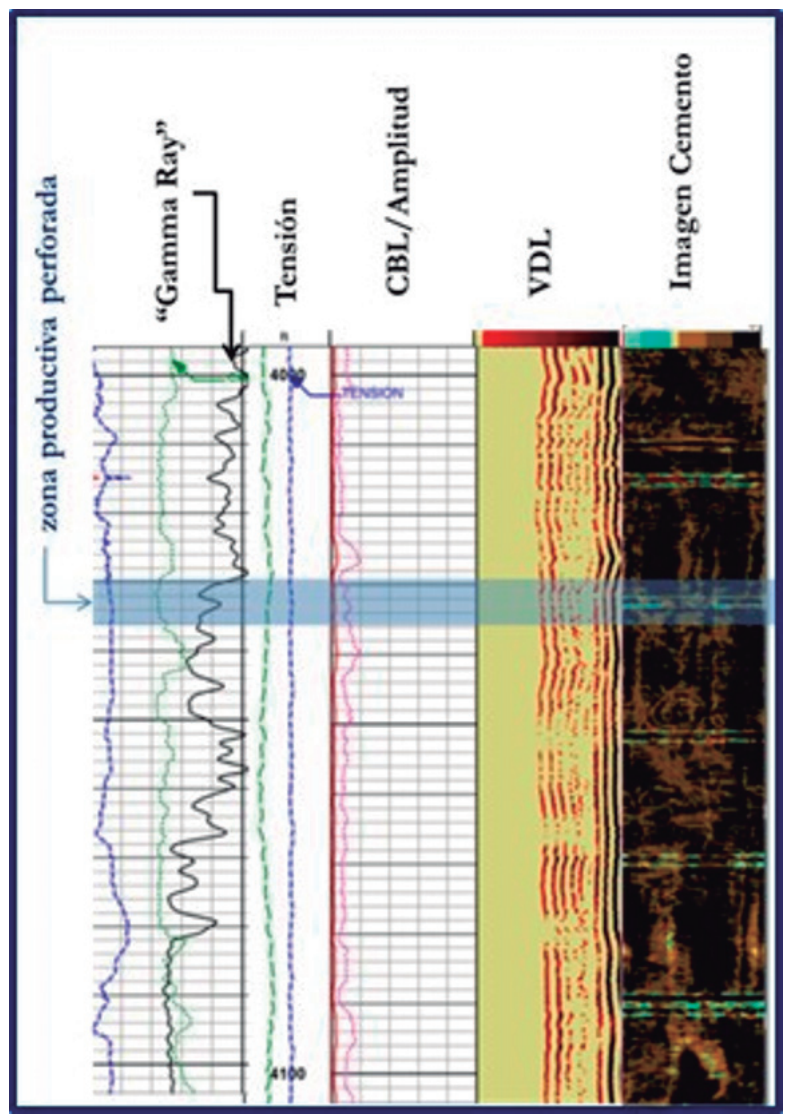

Figura 11. Registro típico compuesto integrado CBL/ VDL en pozo revestido - "cased hole log" (modificado de Halliburton Energy Center, 2001).

\section{f) Análisis con correlaciones estratigráficas}

En este caso se procede a determinar el nivel del contacto de agua-petróleo actual (CAPA) respecto al contacto agua-petróleo original (CAPO) con la mayor exactitud posible y de acuerdo con la información disponible en el campo. Para esta actividad se debe contar con el mapa estructural al tope de la arena, las correlaciones estratigráficas de los pozos ubicados en la misma arena que son objeto de análisis y de algún software comercial que pueda procesar este tipo de información. El objetivo es conocer el nivel actualizado del contacto agua-petróleo, para que posteriormente se pueden sugerir las posibles soluciones técnicas al problema de conificación. Esto disminuye el riesgo de aplicar dicha solución en una zona que está saturada completamente de agua, ya que los métodos deberán ser aplicados por encima del CAPA, de lo contrario, la estrategia implementada puede fracasar.

Del mapa estructural al tope de la arena, objeto de diagnóstico, se determina el CAPO (contacto de aguapetróleo original). El mismo se obtuvo inicialmente de los pozos exploratorios del campo, perforados en esa arena y que mostraron el contacto en el registro eléctrico de hueco abierto durante la campaña de exploración y desarrollo inicial del campo.

Luego de que el campo está desarrollado con varios pozos que producen de la arena objeto de análisis, se procede a generar la correlación estratigráfica entre los pozos ubicados en la arena productora, para lo cual se deberá disponer de algún tipo de registro, preferiblemente eléctrico, pero que muestre los "tracks" con profundidades de TVD SS ("True Vertical Depth Sub Sea"). En la pista ("track") del perfil de resistividad se marca el nivel del CAPO. Posteriormente, se deberá marcar el nivel del CAPA. Para estimar el nivel del CAPA, se procede en dos etapas:

- Se grafica en Log-Log las producciones acumuladas de agua (Wp) versus la de petróleo o $\mathrm{Np}$ (desde el inicio de producción del yacimiento) que contenga la información de todos los pozos productores de la arena objeto del análisis. Si existe algún pozo que está produciendo en conjunto (“commingled”) con otras arenas, se debe excluir de este análisis. Esta premisa es válida para un volumen determinado, es decir, el área de drenaje de los pozos que es común para todos los pozos que han producido en esa arena de forma selectiva.

- Una vez seleccionados los pozos de este análisis, se grafican solo los pozos que pueden producir selectivamente de la arena objetivo y se determina el área de drenaje tomando como base la experticia del analista que maneja el software comercial para tal fin. Finalmente, del gráfico anterior, $\mathrm{Wp}$ versus $\mathrm{Np}$, se obtiene la altura final con la relación $\mathrm{Wp} /$ Área. Se debe enfatizar que este dato representa un estimado, y corresponde a un balance de materiales solo confinado a los pozos bajo estudio. Aunque desarrollar esta actividad es factible, la misma dependerá si se dispone de los pozos de interés correlacionados en la arena objetivo. Sin embargo, es posible encontrarse en proyectos donde no todos los pozos dispongan de registros de pozos.

- En la correlación estratigráfica se anotará el dato estimado del CAPA que corresponde a la altura final o actual del contacto agua-petróleo (en pies), obtenido a partir de lo descrito con anterioridad. La información del CAPA estará en TVD SS, así que luego se deberá trasladar al registro eléctrico del pozo donde se desea solventar el problema de conificación de agua y ese nivel deberá ser anotado en MD o TVD según la preferencia del analista. 
En la Figura 12 se muestra un ejemplo de una correlación entre dos pozos de una misma arena productora que indica el CAPO en 9803' TVD SS y finalmente el estimado del CAPA (usando el método anterior) en 9678' TVD SS. Esta diferencia corresponde a un aumento gradual del contacto de 125 pies. Este se considera el mejor estimado del CAPApara este caso en particular.

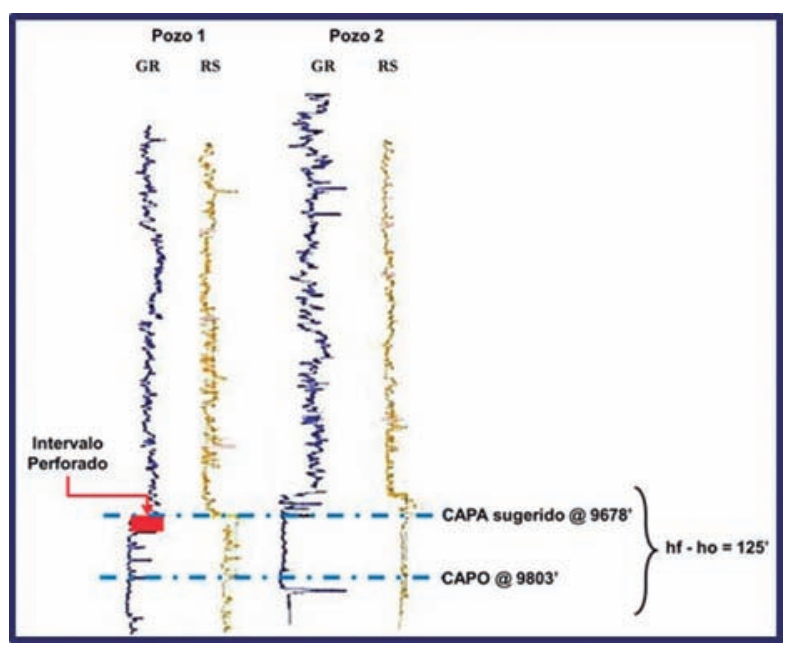

Figura 12. Ejemplo de correlación estratigráfica entre pozos para estimar el CAPA por balance de materiales.

\section{Soluciones al problema de producción excesiva de agua debido al mecanismo de conificación}

El control correctivo de la producción excesiva de agua asociado a un mecanismo de conificación, ha demostrado ser una de las actividades de mayor complejidad y dificultad, especialmente en etapas de producción avanzada de los pozos productores con problemas de conificación. Entre las estrategias que se pueden considerar primeras opciones incluyen:

- Modificación u optimización de los parámetros de producción con el fin de mantener la tasa de producción por debajo de la tasa crítica (máxima tasa con la cual se puede producir petróleo sin llegar a la conificación). Esto incluye optimizar la tasa de producción con el sistema de levantamiento artificial de los fluidos, generalmente bombas del tipo BES (bombeo electro sumergible) o bombas de cavidades progresivas o PCP (del inglés "Progressive Cavity Pumps"). Estos sistemas de levantamiento son capaces de generar una alta caída de presión "drawdown" que pueden acelerar la formación del cono de agua.
- Cerrando el pozo por un período de tiempo, permitiendo el abatimiento del cono formado por mecanismos naturales (p.e. gravedad, gradiente de densidades, etc.) y por ende promover una redistribución de la saturación de los fluidos influenciado por la energía del acuífero de fondo.

Es importante mencionar que el caso de conificación más común y discutidos en el presente trabajo, es cuando el cono proviene de un sistema bajo flujo tipo radial, matricial o cono tridimensional. La existencia de un caso específico que se presenta al cortar una fractura el acuífero activo subyacente, denominado "conificación 2D" (Elphick \& Seright, 1998), no es tema de estudio o análisis del presente artículo.

\section{a) Soluciones Químicas}

El uso de soluciones químicas para el control de la excesiva producción de agua y más específicamente en mecanismos de conificación, ha sido la alternativa más accesible a nivel mundial (Sydansk \& Southwell, 2000) (Jaripatke \& Dalrymple 2010) (El-Karsani, AlMuntasheri \& Hussein, 2014).

Es muy importante resaltar, que tanto para estas soluciones químicas como para cualquier otra descrita a continuación, se hace necesario y casi obligatorio el conocimiento de su ventana de aplicación. Para el caso de las soluciones químicas comercialmente disponibles, dicha ventana se restringe principalmente al conocimiento, lo más preciso posible, de la temperatura de yacimiento ya que es el parámetro que gobierna en la mayoría de los casos las reacciones químicas de estos sistemas en el medio poroso. Adicionalmente, se hace importante conocer cuál es el mecanismo principal de acción por el cual se “corregiría” el cono, ya sea una solución sellante/bloqueante con geles poliméricos fuertes ("strong gels"), una solución con efecto de modificación de la permeabilidad relativa (RPM del inglés "Relative Permeability Modifier") o la inyección de sistemas de geles débiles ("weak gels"). Marín (2018), presenta una actualización a la fecha sobre las alternativas químicas disponibles y que en su mayoría son mezclas o formulaciones de componentes químicos, que interactúan por diferentes mecanismos de reacción. Estas interacciones químicas están influenciadas por las condiciones del yacimiento, muy en especial la temperatura, y la composición del agua de yacimiento).

Para el control correctivo de la conificación de agua, usando este tipo de soluciones químicas, Meyer \& Gardner (1954) desarrollaron un concepto bastante 
simple para tratar un fenómeno de conificación de agua avanzada o en etapas tardías en la vida productiva del pozo productor. Este concepto es conocido como el método de torta, ("pancake" del inglés), Figura 13. En este método se define un radio de aproximadamente 40 pies en la base o a unos pies por encima de la base del cono de agua. Esta simple aproximación es muy común observarla en empresas operadoras para diseñar de manera preliminar un tratamiento químico para el control de la conificación de agua en pozos productores. Fundamentalmente, la mayor limitación de esta aproximación es asumir que la formulación química solo fluirá en la zona de agua o base del cono. Eventualmente, el tratamiento químico fluirá por zonas de menor resistencia al flujo porque dependerá en gran medida de la anisotropía vertical (kv/kh) del yacimiento, así como de las condiciones operacionales del tratamiento (p.e. tasas de inyección). La selección del radio de penetración y su respectivo volumen equivalente, así como la técnica de colocación del "pancake" ha sido muy cuestionada en la literatura (Faber, Joosten, Hashmi \& Gruenenfelder, 1998) (Seright, Lane \& Sydansk, 2001). Adicionalmente, es importante mencionar que el diseño de esta aproximación "pancake" y estimación del radio de penetración de acuerdo a lo sugerido por Meyer \& Gardner (1954), se basan en correlaciones empíricas que han mostrado ser poco confiables.
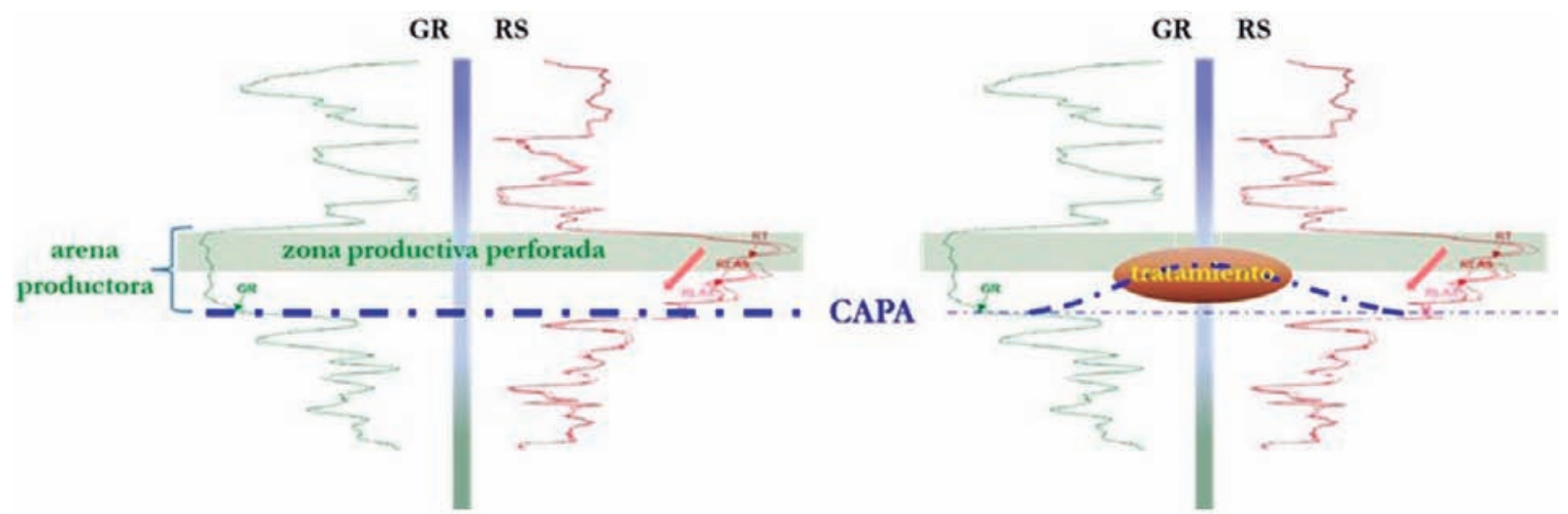

Figura 13. Esquema de un pozo ejemplo para tratamiento químico tipo "pancake" y su colocación.

Por ser condiciones muy específicas, la probabilidad de éxito del uso de tratamientos químicos para la corrección y el control de la conificación de agua en pozos productores ha sido muy baja. Recientes casos históricos se reportan exitosos y proveen lecciones aprendidas en base al uso de tratamientos químicos para el control de la conificación de agua en pozos productores (Hunter, Soetedja, Rinadi, Mitchell \& Gauntt, 1999), (Prado et al., 2005), (Thomas, Shtepani, Marosi, \& Bennion, 2002), (Vega, Campomenosi, Hernández, Moglia \& Peltier, 2018). Por lo tanto y basados en dichas experiencias de tratamientos químicos (p.e. water shut-off), permiten concluir que el uso de tratamientos químicos (p.e. geles fuertes) sigue siendo una tecnología viable para el control de conificación de agua. Sin embargo, el análisis y diagnósticos detallados, el adecuado diseño de operación y en algunos casos el uso de estrategias de inyección no convencionales (p.e. tratamientos en cabeza de pozo "bullheading" por cargas) representan los factores críticos para disminuir la incertidumbre e incrementar la probabilidad de éxito de este tipo de tratamientos.

\section{b) Soluciones Mecánicas}

Las soluciones mecánicas son las de uso más común, y han sido descritas por Sydansk \& Romero-Zerón (2011). Sin embargo, estas tecnologías no son objeto de análisis en el presente trabajo, excepto por dos soluciones mecánicas y otra solución híbrida o alterna para el control y manejo de la conificación de agua en pozos verticales o de poca desviación que serán descritos a continuación.

\section{b.1) Tecnología de Completación Doble}

Una vez que se ha detectado el contacto agua-petróleo actual (CAPA) y se ha diagnosticado el problema de conificación de agua, una de las soluciones mecánicas posibles a evaluar para corregir dicho problema, es la tecnología de completación doble, también conocida como "Downhole Water Sink" o DWS por sus siglas en inglés. (Ramos, 2000). El principio básico de esta estrategia de completación consiste en la producción de forma simultánea de fluidos, el agua de formación del intervalo perforado inferior por encima o cercano del CAPA; y el crudo con bajo corte de agua o incluso sin 
agua, del intervalo perforado superior de la misma arena productora y sin intercalaciones de lutitas (Figura 14). El primer pozo que fue completado utilizando DWS fue en Nebo Hemphill, La Salle Parish, Louisiana (Swisher $\&$ Wojtanowicz, 1995). Este pozo estuvo en producción por más de un año para su evaluación y seguimiento. El agua producida se transportaba directamente hacia las facilidades de superficie del campo para su tratamiento y disposición. A la fecha y a nivel del continente americano, se han evaluado o documentado pocas aplicaciones de esta estrategia de completación en yacimientos con presencia de acuíferos activos. Algunas de las razones por las cuales la completación DWS no haya sido ampliamente considerada, incluyen sin limitarse, a las siguientes:

- Costos elevados de la doble completación con doble equipo de bombeo del tipo electro sumergible, BES (ESP del inglés Electro Submersible Pump),

- Desconocimiento de soluciones mecánicas para conificación, de parte del personal de operaciones en la industria de petróleo y gas de la región,

- Falta del trabajo en equipo entre personal de las empresas de servicio y operadoras.

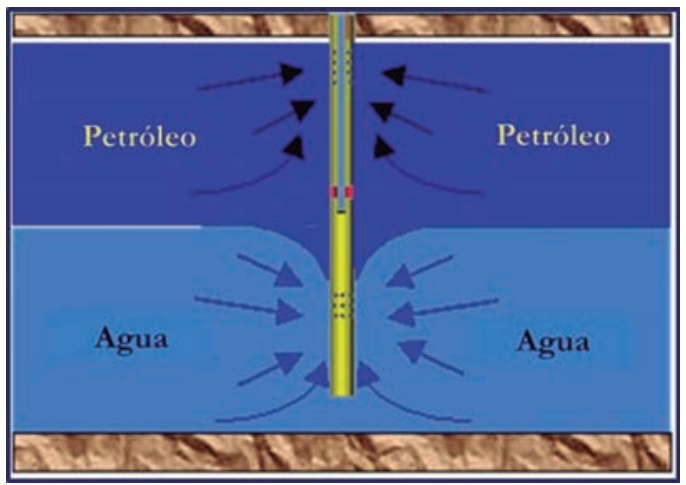

Figura 14. Comportamiento de completación "Downhole Water Sink" (DWS) para control de conificación (Ramos, 2000).

Los autores del presente artículo consideran que entre los factores mencionados, y que han influenciado en el bajo uso de la tecnología de completación DWS, ha sido la poca integración de los equipos de trabajo (p.e. operadoras con empresas de servicio y consultores). Durante la etapa del diagnóstico del pozo candidato, el conocimiento de las propiedades petrofísicas de la zona de interés y la forma en que estas propiedades de la roca se estiman o cuantifican, pueden conducir a la incorrecta selección del pozo idóneo para el uso de la completación DWS. Por otra parte, las empresas de servicio que hacen la instalación en campo de este tipo de completación, por lo general no disponen del personal que sea experto en el área de "Water Conformance", basando sus decisiones exclusivamente desde el punto de vista operacional. Lo anterior representan algunos ejemplos de cómo la combinación de diversos factores, han podido influenciar negativamente con el avance del uso esta tecnología de solución mecánica, para controlar el fenómeno de conificación de agua (Ramos, Shirman, Wojtanowicz, 2000) (Marcano \& Wojtanowicz, 2002). Con relación a costos de la completación DWS, en aquellos casos en que el operador disponga de gas de levantamiento en el campo (“Gas Lift”), es posible diseñar la doble completación con un sistema de doble levantamiento artificial por gas ("Dual Gas Lift"), con el fin de reducir costos de inversión en comparación a las completaciones que disponen de dos bombas electro sumergibles o "Dual ESP" (Marcano \& Wojtanowicz, 2002).

\section{b.2) Tecnología de Separación de Fondo}

Otra opción mecánica para el control de alta producción de agua incluyendo problemas de conificación a nivel de pozo, es la separación dinámica de fondo con hidrociclones, o mejor conocida como "Downhole Oil Water Separation” (DOWS o DHOWS por sus siglas en inglés). La completación incluye una a dos etapas de separación aceite-agua. A mayor número de etapas, mayor será la eficiencia de separación del agua. La instalación de estos sistemas puede considerar dos opciones:

- producir agua y aceite con menor contenido de agua por separado o,

- producir aceite con menor contenido de agua y reinyectar el agua separada en el mismo yacimiento o en otros intervalos/yacimientos de la columna estratigráfica.

Esta tecnología mostro su auge en la década de los 90's, pero según las estadísticas de Departamento de Energía de los Estados Unidos de América (U.S: DOE, del inglés United States Department of Energy), para finales del año 1999 solamente se contaban con cien instalaciones de DOWS a nivel mundial. Esto es debido a limitaciones técnicas y económicas para la implementación de esta tecnología (Bangash \& Reyna, 2003). A nivel técnico y de forma similar que ha sucedido con las completaciones DWS, se sospecha de una mala selección de los pozos preseleccionados para la instalación de esta tecnología. Por lo tanto, es muy importante tener un buen conocimiento de las propiedades petrofísicas y anisotropía vertical, entre otras variables, del pozo candidato para hacer la selección adecuada de completaciones mecánicas para 
el control de agua en las cercanías del pozo productor. Adicionalmente, se debe contar con la experiencia en técnicas de control de agua "Water Conformance" que por lo general empresas operadoras se ven forzadas a delegar esta actividad a terceros en vista de la poca disponibilidad de expertos en la materia a nivel internacional. Con relación a costos, en aquellos yacimientos donde hay grandes reservas remanentes, es necesario hacer el estudio de factibilidad económica a fin de justificar la aplicación de la tecnología DOWS. Sin embargo, para justificarse el estudio de factibilidad técnica y económica se debe disponer de datos confiables del sistema pozo-yacimiento-tecnología DOWS. La selección del pozo no se puede hacer por ensayo y error, como posiblemente ha ocurrido en el pasado. Otra posibilidad del bajo uso de esta tecnología puede deberse a diferentes factores entre los cuales resalta el uso de esta completación en fase de madurez muy avanzadas (p.e. pozos marginales) haciendo difícil su justificación económica. Otra limitación puede ser la selección de la reinyección del agua separada a fondo de pozo, ya que la misma no necesariamente cumple con la calidad de agua suficiente (p.e. contenido de grasas y sólidos) para ser reinyectada sin que ocurra una pérdida de inyectividad en las zonas seleccionadas incrementándose los costos de la misma.

\section{b.3) Solución híbrida o alterna}

A continuación se procederá a resumir la tecnología AWACT ("Anti Water Coning Technology") y que en el presente artículo se sugiere como una propuesta de solución híbrida al problema de conificación de agua en yacimientos y/o pozos con acuíferos de fondo.

Los efectos de histéresis de la permeabilidad relativa son la base de la tecnología AWACT, patentada por AOSTRA ("Alberta Oil Sands Technology Research Authority" por sus siglas en inglés) en la década de los 80’s (Luhning, Chmilar \& Anderson, 1990), (Bennion, Thomas \& Bietz, 1996) y (Meneses, Moreno, \& Plata, 2017). En la región geográfica de Alberta, Canadá, la producción primaria de los yacimientos de crudos pesados era soportada por el empuje por acuíferos activos. Debido a la relación de movilidad desfavorable entre las fases aceite-agua y características de las formaciones productoras (p.e. anisotropía vertical en el área de drenaje de los pozos productores), la conificación de agua se evidenciaba con regularidad llegándose a cerrar los pozos por alcanzar su límite económico. Durante la década del desarrollo de la tecnología AWACT, se disponía de gas $\mathrm{CO}_{2}$ en campos cercanos a los yacimientos donde se evidenciaba el problema de conificación de agua. Ese recurso local, fue aprovechado para hacer las inyecciones cíclicas de gas en a pozos con altas RAP (WOR). El mecanismo para ese proceso cíclico consta de formación de una capa de gas adicional que empuja los fluidos ubicados en el ático o tope de la arena productora, hacia los canales de alta permeabilidad. Con la reactivación de estos canales, la producción del pozo mostrara una condición de gas atrapado que genera una reducción de la permeabilidad al agua y en consecuencia, una redistribución del flujo de fluidos en la formación. Es decir, la presencia de gas en la formación logra modificar el perfil de saturaciones trifásica, por encima del CAPA. Específicamente, en pozos con problemas de conificación de agua se apreciará una disminución considerable de la RAP (NEXEN INC., 2013). La RAP disminuirá en el tiempo, a medida que se inyecten nuevos ciclos de gas en el pozo productor. Esta aplicación particular, es específica para casos de "Water Conformance", y es por eso que se considera un método híbrido, al combinar conceptos de recobro mejorado (p.e. procesos de inyección alternada de agua y gas inmiscible) y métodos de control de agua o "conformance".

La inyección cíclica de $\mathrm{CO}_{2}$ referida usualmente como un proceso de "Huff and Puff" (similar a la inyección cíclica de vapor), en condiciones inmiscibles, consta de la inyección de un volumen determinado de gas en un pozo productor. Una vez inyectado el volumen de gas el pozo ("Huff") se cierra por un periodo de remojo ("Puff") establecido generalmente por simulaciones numéricas en modelos radiales de un solo pozo. Cumplido el período de remojo el pozo es reabierto a producción. En ciertos yacimientos de crudos livianos los resultados han sido exitosos y se ha observado una vida promedio del tratamiento que varía de uno hasta cuatro años (Miller, 1990). El éxito de esta técnica está relacionado con diferentes variables que se resumen a continuación:

- el volumen de gas inyectado en condiciones inmiscibles en el área de drenaje de pozo,

- el tiempo de remojo,

- las características de interacción entre el gas de inyección y el petróleo residual,

- el número de ciclos inyectados.

En la Figura 15 se aprecia la curva de permeabilidad relativa agua-aceite con y sin el efecto de histéresis debido a la presencia o no de gas libre en el sistema. Este efecto se observó posterior a la inyección de un ciclo de gas, a condiciones $\mathrm{P}$ y $\mathrm{T}$ de laboratorio y con la premisa que el medio poroso es humectado por agua. Hay que enfatizar que el efecto de histéresis de la permeabilidad relativa es producto de una saturación de gas atrapado 
(característico para cada medio poroso), generado por la inyección cíclica de gas, sea $\mathrm{CO}_{2}$ o cualquier otro disponible.

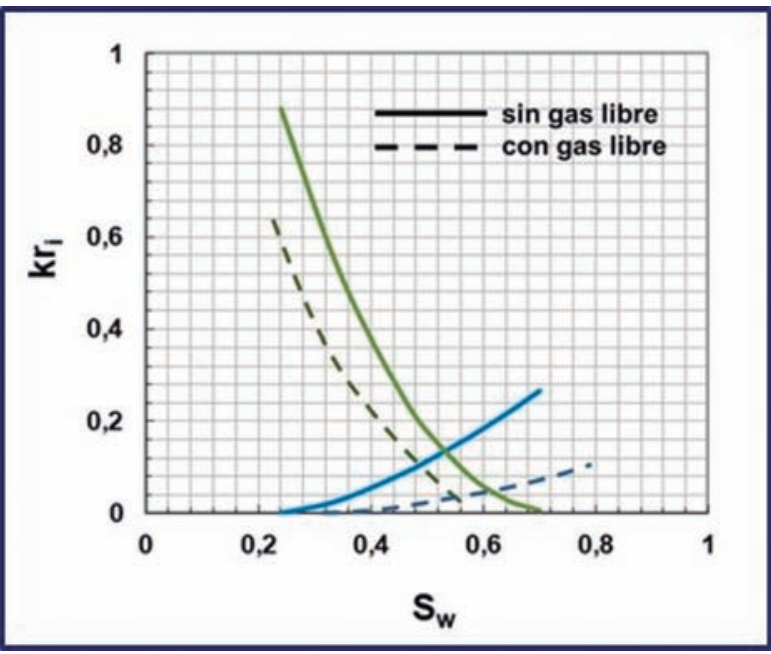

Figura 15. Efecto de saturación de gas libre en curva de permeabilidad relativa, con histéresis, tecnología AWACT (modificado de Bennion, 1996).

Respecto a la selección de pozos candidatos y criterios para el uso de la tecnología AWACT, no será discutida en el presente trabajo. Sin embargo, se hace énfasis en el potencial de la aplicación de esta tecnología como posible estrategia para controlar el problema de conificación de agua.

De manera similar a lo discutido con anterioridad respecto a las soluciones mecánicas, se presentó una disminución importante del uso de la tecnología AWACT en Canadá después de los 90’s. Los autores consideran que las posibles razones, son una combinación de factores tales como:

- Uso del gas de $\mathrm{CO}_{2}$ para proyectos de recuperación mejorada, y en proyectos aguas abajo (“downstream”),

- Costosa implementación de las unidades generadoras de nitrógeno portátiles y/o a gran escala,

- Escasez del gas natural asociado a pozos productores, que luego sería utilizado principalmente para proyectos de levantamiento artificial por gas ("gas lift”).

- Expansión de proyectos de SAGD ("Steam Assisted Gravity Drainage”) a partir del año 2000, entre otros proyectos de recuperación mejorada por métodos químicos (p.e. polímeros y álcali-surfactantepolímero).
- Volatilidad de los precios del petróleo, entre otras.

Por lo expuesto anteriormente, los autores sugieren varios escenarios a considerar, en caso de encontrar los pozos candidatos idóneos para el control de conificación con la técnica de AWACT y disponer de fuentes de gas a costos razonables:

- De disponerse de un excedente del gas comprimido para el sistema de levantamiento artificial ("gas lift”) u otro gas no condensable, se puede usar perfectamente para este propósito basados en los resultados reportados por NEXEN INC. (2013) (Figura 16),

- Adquirir o contratar servicio de unidad generadora de $\mathrm{N}_{2}$ portátil,

- Inyección de gases de combustión $\left(\mathrm{CO}_{2}\right.$ y $\left.\mathrm{N}_{2}\right)$ generados a partir de sistemas comerciales que usen corrientes de gas natural, carbón o combustibles líquidos.

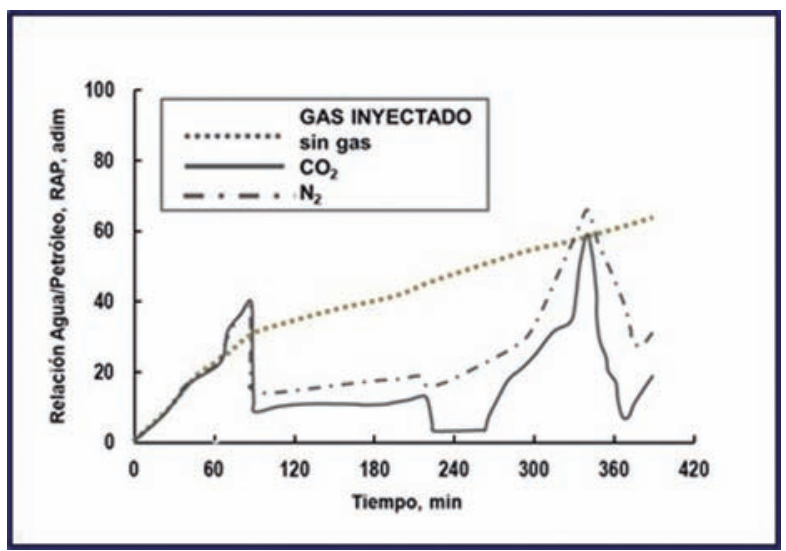

Figura 16. Impacto del tipo de gas inyectado sobre la relación agua-petróleo (modificado de NEXEN INC. 2013).

Finalmente, es muy importante destacar que las soluciones u opciones propuestas, generalmente requieren de una inversión inicial importante. Por lo tanto, se recomienda hacer un estudio conceptual y de factibilidad técnico-económica, con el fin de validar la justificación de evaluar la tecnología a escala piloto o previa a una potencial expansión a nivel de campo.

\section{Conclusiones}

1. El mecanismo de conificación de agua, se presentan muy comúnmente en pozos productores de petróleo y gas en yacimientos con acuíferos de fondo, donde se requiere de un cuidadoso y adecuado plan de estudio de diagnóstico y de propuesta de solución. 
2.Las metodologías de diagnósticos presentadas, basadas en la información básica de uso rutinario, tales como gráficas de históricos de producción y de diagnóstico tipo Chan y Ramos, registros GR/RS ("gamma ray"/ resistividad) y correlaciones estratigráficas entre pozos vecinos, pueden determinar la presencia de un mecanismo de conificación de agua. Esto se presenta en el trabajo como un plan o proceso que se ha denominado Revisión, Análisis e Interpretación (RAI).

3.La herramienta de diagnóstico usando las correlaciones estratigráficas entre pozos vecinos presentadas en este trabajo, permite identificar el contacto agua-petróleo original (CAPO) y sugerir un contacto de agua actual (CAPA) que ayuda al adecuado diagnóstico del mecanismo de conificación de agua.

4.El uso e implementación de las soluciones presentadas y sugeridas en este trabajo, deberá ser soportado por un estudio conceptual, tomando en consideración su ventana de aplicación y además por una evaluación técnico-económica. Esto con la finalidad, de validar si se justifica la aplicación de la solución a escala piloto y como paso previo a una potencial expansión a nivel de campo.

\section{Referencias}

1. Bailey, B., Crabtree, M., Tyrie, J., Elphick, J., Kuchuck, F., Romano, C. \& Roodhart, L. (2000). Water Control. Oilfield Review, (12), 30-51.

2. Bangash, Y. K. \& Reyna, M. (2003). Downhole Oil Water Separation (DOWS) Systems in High Volume/High HP Application. (SPE 81123). SPE Latin American and Caribbean Petroleum Engineering Conference, Port of Spain, Trinidad and Tobago.

3. Bennion, B. D., Thomas, B. F. \& Bietz, R. F. (1996). Hysteretic Relative Permeability Effects and Reservoir Conformance, An Overview. (P0002-96). PNEC International Conference on Reservoir Conformance, Profile Control, Water and Gas Water Shut Off, Houston, USA.

4. Chan, K. S, Bond, A. J., Keese, R. F. \& Lai, Q. J. (1996). Diagnostic Plots Evaluate Gas Shut-Off Gel Treatments at Prudhoe Bay, Alaska (SPE 36614). SPE Annual Technical Conference and Exhibition, Denver, USA.

5. Chan, K. S. (1995). Water Control Diagnostic Plots. (SPE 30775). SPE 70th Annual Technical Conference and Exhibition, Dallas, USA.
6. Chaperon I. (1996). Theoretical Study of Coning Towards Horizontal and Vertical Wells in Anisotropic Formations: Subcritical and Critical Rates. (SPE 15377). SPE Annual Technical Conference and Exhibition, New Orleans, Louisiana, USA.

7. Economides, M., Watters, L., Dunn-Norman, Hardy S., Lockhart, T. (1998). Petroleum Well Construction: Water Control. New York, USA: John Wiley \& Sons.

8. El-Karsani, K. S.M., Al-Muntasheri, G.A., \& Hussein, I. A. (2014). Polymer Systems for Water Shutoff and Profile Modification: A Review Over the Last Decade. (SPE 163100PA). SPE Journal, V19 (1), February.

9. Elphick, J.E., \& Seright, R.S. (1998). AClassification of Problem Water Types. PNEC 3rd International Conference on Reservoir Conformance, Profile Control, Water and Gas Shutoff, Houston, TX.

10. Faber, M. J., Joosten, G. J., Hashmi K. A, \& Gruenenfelder, M. (1998). Water shut-off field experience with a relative permeability modification system in the Marmul field (Oman). (SPE-39633). SPE/DOE Improved Oil Recovery Symposium, Tulsa, USA.

11. Halliburton Energy Center, (2001). Conformance Technology. Identification and Treatment of Water Control Problems for Improved Reservoir Recovery Efficiency. (Publication F-3373). Houston, USA.

12. Hardy, M., \& Lockhart, T. (1998). Petroleum Well Construction: Water Control. New York, USA. John Wiley \& Sons Ed.

13. Hunter, S., Soetedja, V., Rinadi, M., Mitchell, W., \& Gauntt, G. (1999). Successful Application of Water-Conformance Technology: A Case Study from East Kalimantan, Indonesia. (SPE 54352). SPE Asia Pacific Oil and Gas Conference and Exhibition, Jakarta, Indonesia.

14. Jaripatke, O. \& Dalrymple, D. (2010). WaterControl Management Technologies: A Review of Successful Chemical Technologies in the Last Two Decades. (SPE 127806). SPE International Symposium and Exhibition on Formation Damage Control, Lafayette, USA. 
15. Khatib, Z. \& Verbeek, P. (2002). Water to Value Produced Water Management for Sustainable Field Development of Mature and Green Field. (SPE 73853). SPE International Conference on Health, Safety, and Environment in Oil and Gas E\&P, Kuala Lumpur, Malaysia.

16. Kuo M. C. T. \&, DesBrisay C. L. (1983). A Simplified Method for Water Coning Predictions. (SPE 12067). SPE Annual Technical Conference and Exhibition, San Francisco, California, USA.

17. Kuo, M. C. T. (1989). Correlations rapidly analyze water coning. Oil \& Gas Journal, Oct. 2. pp. 77-80.

18. Llamedo, M., Mejías, F., González, E. R., Espinoza, J. \& Valero, E. M. (2005). Successful Gas Shutoff with Gel, Evaluation and Implementation, North East, Venezuela. (SPE 96696). SPE Offshore Europe, Aberdeen, Scotland.

19. Luhning, R.W., Chmilar M. J. \& Anderson, B. S. (1990). The AOSTRA Anti Water Coning Technology (AWACT) Process - From Invention To Commercial Application. (PETSOC-90-132). PSC Annual Technical Meeting, Calgary, Canada.

20. Marcano, L. \& Wojtanowicz, A. K. (2002). Dual Gas Lift in Wells with Downhole Water Sink Completion. (PETSOC-2002-238). Canadian International Petroleum Conference, Calgary, Canada.

21. Marín, A. (2018). WSO (Water Shut-Off) Lab \& Field Connection. Workshop Nuevas Tendencias Tecnológicas para Control de Agua en Fondo. Frontera Energy, Bogotá, Colombia.

22. Martin, C. A. G., \& Páez, E. G. M. (2017). Efeito da salinidade na tensão interfacial do sistema óleo/ agua em condições isobáricas e incremento gradual da temperatura. Fuentes: El reventón energético, 15(2), 117-124.

23. Meneses, A. F. O., Moreno, L. F. C., \& Plata, J. A. R. (2017). Metodología experimental para la estimación de permeabilidades relativas en dos y tres fases por medio de ajuste histórico. Revista Fuentes, 15(1), 75-85. https://revistas. uis.edu.co/index.php/revistafuentes/article/ view/6504
24. Meyer, H., \& Gardner, A. (1954). Mechanics of Two Immiscible Fluids in Porous Media. Journal of Applied Physics, 25, 1400-1406.

25. Miller, B. J. (1990). Design and Results of a Shallow, Light Oilfield Wide Application of $\mathrm{CO}_{2}$ Huff 'n' Puff Process. (SPE 20268). SPE/ DOE Enhanced Oil Recovery Symposium, Tulsa, USA.

26. Muskat, M. (1949). Physical Principles of Oil Production. New York, USA, McGraw-Hill Book Col. Inc.

27. NEXEN INC., Calgary (2013). Steam anti-coning/ cresting technology (SACT) remediation process. Kelso Kerr, Richard; Yang, Peter. U.S. Cl. E2IB 43/24 (2013.01), US 2013/0284461 A1. October 21.

28. Prado, M., Palencia, J., Reyna, M., Fernández, I., Espinoza, J. \& Rauseo, O. (2005). Two Different Water Shutoff Applications in a Poorly Consolidated Sandstone Reservoir with Strong Waterdrive. (SPE 93060). SPE International Symposium on Oilfield Chemistry, The Woodlands, USA.

29. Ramos, L., Shirman, E. \& Wojtanowicz, A. K. (2000). Vertical Interference Testing Method using Dual Completions with Downhole Water Sink. (SPE 62921). SPE Annual Technical Conference and Exhibition, Dallas, USA.

30. Ramos, L. (2000). An Assessment Method for Field Implementation of the Downhole Water Sink "DWS" Technology. Master of Science in Petroleum Engineering Thesis. Louisiana State University, Louisiana, USA.

31. Recham, R., Osisanya, S.O., \& Touami M. (2000). Effects of Water Coning on the Performance of Vertical and Horizontal Wells-A Reservoir Simulation Study of Hassi R'mel Field, Algeria. (SPE 65506). International Conference on Horizontal Well Technology, Calgary, Canada.

32. Reyes, C. M., Ortiz, J. L. V. \& Azuaje, E. M. (2010). A Reliability Based Systemic Method for Water Production Analysis, Diagnosis and Solution Design. (SPE 138935). SPE Latin American and Caribbean Petroleum Engineering Conference, Lima, Peru. 
33. Satter, A., Thakur, G. C. (1994). Integrated Petroleum reservoir Management. Tulsa, OK, United States. PennWell Publishing Company.

34. Seright, R. S., Lane, R. H., \& Sydansk R. D. (2001). A Strategy for Attacking Excess Water Production. (SPE 70067). SPE Permian Basin Oil and Gas Recovery Conference, Midland, USA.

35. Seright, R. S. (1997). Improved Methods for Water Shutoff. Final Report, DOE/BC/91008-4, U.S. DOE.

36. Soliman, M. Y., Rester, S., Sigal, R., Everett, D. \& Johnson, M. H. (2000). Integration of Technology Supports Preventive Conformance Reservoir Techniques. (SPE 62553). 2000 SPE/AAPG Western Regional Meeting, Long Beach, USA.

37. Soliman, M., East, L. \& Gorel, S. (1999). Reservoir Conformance Approach and Management Practices for Improved recovery Opportunities: Process and Case History. (SPE 53918). SPE Latin American and Caribbean Petroleum Engineering Conference, Caracas, Venezuela.

38. Swisher, M.D. \& Wojtanowicz, A.K. (1995). New Dual Completion Method Eliminates Bottom Water Coning. (SPE 30697). SPE Annual Technical Conference and Exhibition, Dallas, USA.

39. Sydansk, R. \& Romero-Zerón, L. (2011). Reservoir Conformance Improvement. Texas, USA: Society of Petroleum Engineers.
40. Sydansk, R. D. \& Southwell, G. P. (2000). More than 12 Years' Experience with a Successful Conformance-Control PolymerGel Technology. (SPE 62561). SPE/AAPG Western Regional Meeting, Long Beach, USA.

41. Thakur, G. C., Satter, A. (1998). Integrated Waterflood Asset Management. Tulsa, OK, United States, PennWell Corporation.

42. Thomas, F. B., Shtepani, E., Marosi, G. \& Bennion, D. B. (2002). Production Well Water Coning-Is There Anything We Can Do? (PETSOC-2002-031). Petroleum Society's Canadian International Petroleum Conference, Calgary, Canada.

43. Vega, I. N., Campomenosi, A., Hernández, M. I., Moglia, D. \& Peltier, J. (2018). Successful Water Shut-Off Treatment Specially Designed for High Temperature: Vizcacheras Experience. (SPE 190156). SPE Improved Oil Recovery Conference, Tulsa, USA.

44. Velandia, J. L. P. (2013). Simulación numérica del flujo bifásico agua-petróleo en un medio poroso. Fuentes: El reventón energético, 11(2), 10.

45. Zabala, R., Coral, D., Ramírez, A., Rojas, R., Solarte, H., Medina, S., Ruiz, M. (2017). Un Modelo de Diagnóstico del Impacto de la Producción de Agua en Pozos Productores de Petróleo. Congreso Colombiano del Petróleo ACIPET, Bogotá D.C., Colombia.

Recepción: 24 septiembre de 2018

Aceptación: 26 de noviembre de 2018 